\title{
ON SIMULTANEOUS PELL EQUATIONS AND RELATED THUE EQUATIONS
}

\author{
BO HE, ÁKOS PINTÉR AND ALAIN TOGBÉ
}

\begin{abstract}
In this paper, we prove that the simultaneous Pell equations$$
x^{2}-\left(m^{2}-1\right) y^{2}=1, z^{2}-\left(n^{2}-1\right) y^{2}=1
$$

have only positive integer solution $(x, y, z)=(m, 1, n)$ if $m<n \leq m+m^{\varepsilon}, 0<$ $\varepsilon<1$ and $m \geq 202304^{\frac{1}{1-\varepsilon}}$. Using a computational reduction method we can omit the lower bound for $m$ when $m<n \leq m^{\frac{1}{5}}$. Moreover, we apply our main result to a family of Thue equations in two parameters studied by Jadrijević $[?]-[?]$.
\end{abstract}

\section{INTRODUCTION}

Let $a$ and $b$ be distinct non-square positive integers. There is a long history of the simultaneous Pell equations

$$
x^{2}-a y^{2}=1, z^{2}-b y^{2}=1 .
$$

This system of equations plays a crucial role in the classical theory of the figurate numbers and the modern approach of congruent number problem as well, see [?] and [?], respectively. By the work of Thue [?] and Siegel [?], the number of positive integer solutions $(x, y, z)$ of (??) is finite. The first absolute upper bound for the number of solutions to (??) was given by Schlickewei [?], later Masser and Rickert [?] improved his exponential bound to 16. In 1998, combining with simultaneous diophantine approximation and the theory of linear forms in logarithms, Bennett [?] showed that there are at most 3 positive integer solutions $(x, y, z)$ to the system of equations (??). Yuan [?] and [?] proved that for sufficiently large $a$ and $b$, the number of solution is at most two. The best known bound is due to Bennett, Cipu, Mignotte and Okazaki [?]. In fact, they proved that Yuan's result is true for every value of $a$ and $b$.

As it was pointed out by Bennett (see [?]), if the system of equations (??) has positive integer solutions, then it is equivalent to

$$
x^{2}-\left(m^{2}-1\right) y^{2}=1, z^{2}-\left(n^{2}-1\right) y^{2}=1, \quad 1<m<n .
$$

${ }^{1} 2010$ Mathematics subject Classification: 11D09, 11D45, 11B37, 11J86.

Date: July 31, 2014.

The first author were supported by Natural Science Foundation of China (Grant No. 11301363), and Sichuan provincial scientific research and innovation team in Universities (Grant No. 14TD0040), and the Natural Science Foundation of Education Department of Sichuan Province (Grant No. 13ZA0037 and No. 13ZB0036). The second author was supported in part by the Hungarian Academy of Sciences, OTKA grants K100339, NK101680, NK104208 and by the European Union and the European Social Fund through project Supercomputer, the national virtual lab (grant no.: TAMOP-4.2.2.C-11/1/KONV-2012-0010). 
Bennett [?] proved that there are exactly two positive integer solutions of (??) when

$$
n=\frac{\left(m+\sqrt{m^{2}-1}\right)^{2 l}-\left(m-\sqrt{m^{2}-1}\right)^{2 l}}{4 \sqrt{m^{2}-1}},
$$

with $l \geq 2$ and $m \geq 2 \cdot 10^{7} \sqrt{l} \log ^{2} l$. Yuan [?] made the following conjecture.

Conjecture 1. Apart from when $n$ has the form in (??), there is at most one positive integer solution $(x, y, z)$ to (??).

Le [?] showed that the conjecture is true if $m$ and $n$ are sufficiently large and have sufficiently large common divisor. In this paper, we will verify the conjecture when the difference of $n$ and $m$ is small. More precisely, we prove the following result.

Theorem 1. If $m<n \leq m+m^{\varepsilon}$, for some real number $\varepsilon \in(0,1)$, then the simultaneous Pell equations (??) have only the positive integer solution $(x, y, z)=$ $(m, 1, n)$ for $m>202304^{\frac{1}{1-\varepsilon}}$.

When the constant $\varepsilon$ is small, we have

Theorem 2. If $m<n \leq m+m^{\frac{1}{5}}$, then the simultaneous Pell equations (??) possess the unique positive integer solution $(x, y, z)=(m, 1, n)$.

For the organization of this paper, we will use the gap principle to prove some lemmas in Section ??. By the means of a linear form in two logarithms, we prove Theorem ?? in Section ??. We use the reduction method of Baker-Davenport [?] is in Section ?? to solve the system of Pell equations (??) for the small values of $m, n$, therefore we complete the proof of Theorem ??. In the last section, we apply our main result to a family of Thue equations in two parameters studied by Jadrijević [?]-[?].

\section{Gap PRINCIPLeS}

We will rewrite the equations into an equality of two Lucas numbers. We have $y=U_{j}=W_{k}$, i.e.

$$
y=U_{j}=\frac{\alpha^{j}-\alpha^{-j}}{2 \sqrt{a}}=\frac{\beta^{k}-\beta^{-k}}{2 \sqrt{b}}=W_{k},
$$

where $j, k$ are odd positive integers, $\alpha=m+\sqrt{m^{2}-1}, \beta=n+\sqrt{n^{2}-1}$ are the fundamental solutions to the equations $x^{2}-a y^{2}=1$ and $z^{2}-b y^{2}=1$ respectively, with $a=m^{2}-1$ and $b=n^{2}-1$.

In this section, we will consider the following linear form in logarithms

$$
\Lambda=j \log \alpha-k \log \beta+\log \left(\sqrt{\frac{b}{a}}\right) .
$$

Lemma 1. If $j, k>1$, then $j>k$. 
Proof. Since $U_{j}=\frac{\alpha^{j}-\alpha^{-j}}{2 \sqrt{a}}$, by mathematical induction one can show that $\alpha^{j-1}<$ $U_{j}<(2 m)^{j-1}$, for $j>1$. Similarly, we have $\beta^{k-1}<W_{k}<(2 n)^{k-1}$, for $k>1$. From $U_{j}=W_{k}$, we get $\beta^{k-1}<(2 m)^{j-1}$. Since $n \geq m+1$, we have $\beta=$ $n+\sqrt{n^{2}-1} \geq 2 n-1 \geq 2 m+1$. This implies $(2 m+1)^{k-1}<(2 m)^{j-1}$ and then $k<j$.

Lemma 2. If equation (??) holds with $k \geq 1$, then $0<\Lambda<\frac{\alpha^{2}}{\alpha^{2}-1} \cdot \alpha^{-2 j}$.

Proof. One can verify that $\alpha^{j}<\beta^{k}$. Indeed, if $\alpha^{j}>\beta^{k}$, then $\alpha^{j}-\alpha^{-j}>\beta^{k}-\beta^{-k}$. Since $m<n$, then we have $\frac{\alpha^{j}-\alpha^{-j}}{\sqrt{m^{2}-1}}>\frac{\beta^{k}-\beta^{-k}}{\sqrt{n^{2}-1}}$. This contradicts (??). Therefore, we get

$$
0<\Lambda=\log \left(\frac{1-\beta^{-2 k}}{1-\alpha^{-2 j}}\right)<-\log \left(1-\alpha^{-2 j}\right)<\frac{\alpha^{2}}{\alpha^{2}-1} \cdot \alpha^{-2 j}
$$

Lemma 3. If $k>1$, then $(j-1) \log \alpha<(k-1) \log \beta$.

Proof. The condition $k>1$ and Lemma ?? give $j>1$. By Lemma ??, we have $\Lambda<\frac{1}{\left(\alpha^{2}-1\right) \alpha^{2}}$. Since

$$
\begin{aligned}
& \alpha \sqrt{b}-\beta \sqrt{a}=(m+\sqrt{a}) \sqrt{b}-(n+\sqrt{b}) \sqrt{a}=m \sqrt{b}-n \sqrt{a} \\
= & \frac{m^{2}\left(n^{2}-1\right)-n^{2}\left(m^{2}-1\right)}{m \sqrt{b}+n \sqrt{a}}=\frac{n^{2}-m^{2}}{m \sqrt{b}+n \sqrt{a}} \geq \frac{(m+1)^{2}-m^{2}}{m \sqrt{b}+n \sqrt{a}}>\frac{2 m+1}{2 m \sqrt{b}}>\frac{1}{\sqrt{b}},
\end{aligned}
$$

we obtain

$$
\begin{gathered}
(j-1) \log \alpha-(k-1) \log \beta=\Lambda+\log \left(\frac{\beta \sqrt{a}}{\alpha \sqrt{b}}\right)<\Lambda+\frac{\beta \sqrt{a}-\alpha \sqrt{b}}{\alpha \sqrt{b}}<\Lambda-\frac{1}{\alpha b} \\
<\frac{1}{\left(\alpha^{2}-1\right) \alpha^{2}}-\frac{1}{\alpha b}=\frac{b-\left(\alpha^{2}-1\right) \alpha}{\left(\alpha^{2}-1\right) \alpha^{2} b}<0 .
\end{gathered}
$$

The last inequality is easy to get as $\alpha=m+\sqrt{m^{2}-1}>2 \sqrt{m^{2}-1}$ and $n<$ $2 m$.

Let $\Delta=j-k$. Then we have.

Lemma 4. If $k>1, m \geq 8$, then $k-1 \geq 0.99 \Delta m^{1-\varepsilon} \log \alpha$.

Proof. By Lemma ??, we have $\frac{j-1}{k-1}<\frac{\log \beta}{\log \alpha}$. This implies

$$
\begin{aligned}
\frac{\Delta}{k-1} & <\frac{\log \beta}{\log \alpha}-1=\frac{\log (\beta / \alpha)}{\log \alpha}=\frac{\log (1+(\beta-\alpha) / \alpha)}{\log \alpha}<\frac{\beta-\alpha}{\alpha \log \alpha} \\
& =\frac{n+\sqrt{b}-m-\sqrt{a}}{\alpha \log \alpha}=\frac{n-m+\frac{b-a}{\sqrt{b}+\sqrt{a}}}{\alpha \log \alpha}=\frac{(n-m)\left(1+\frac{n+m}{\sqrt{b}+\sqrt{a}}\right)}{(m+\sqrt{a}) \log \alpha} \\
& <\frac{m^{\varepsilon}\left(1+\frac{m}{\sqrt{m^{2}-1}}\right)}{1.99 m \log \alpha} .
\end{aligned}
$$

Therefore, we have

$$
k-1>\Delta \frac{1.99 m^{1-\varepsilon}}{1+\frac{m}{\sqrt{m^{2}-1}}} \log \alpha>0.99 \Delta m^{1-\varepsilon} \log \alpha .
$$




\section{LINEAR FORMS IN TWO LOGARITHMS}

Now we recall the following result due to Laurent (see [?], Corollary 2, page 328 ) on linear forms in two logarithms. For any non-zero algebraic number $\gamma$

of degree $d$ over $\mathbb{Q}$, whose minimal polynomial over $\mathbb{Z}$ is $a \prod_{j=1}^{d}\left(X-\gamma^{(j)}\right)$, we denote by

$$
h(\gamma)=\frac{1}{d}\left(\log |a|+\sum_{j=1}^{d} \log \max \left(1,\left|\gamma^{(j)}\right|\right)\right)
$$

its absolute logarithmic height.

Lemma 5. Let $\alpha_{1}$ and $\alpha_{2}$ be multiplicatively independent, each of $\alpha_{1}, \alpha_{2}, \log \alpha_{1}$ and $\log \alpha_{2}$ is real and positive. $b_{1}$ and $b_{2} \in \mathbb{Z}^{+}$and

$$
\Lambda=b_{2} \log \alpha_{2}-b_{1} \log \alpha_{1} .
$$

Let $D:=\left[\mathbb{Q}\left(\alpha_{1}, \alpha_{2}\right): \mathbb{Q}\right] /\left[\mathbb{R}\left(\alpha_{1}, \alpha_{2}\right): \mathbb{R}\right]$, for $i=1,2$ let

$$
\log A_{i} \geq \max \left\{h\left(\alpha_{i}\right), \frac{\left|\log \alpha_{i}\right|}{D}, \frac{1}{D}\right\}
$$

and

If $|\Lambda| \neq 0$, then we have

$$
b^{\prime} \geq \frac{b_{1}}{D \log A_{2}}+\frac{b_{2}}{D \log A_{1}} .
$$

$$
\log |\Lambda| \geq-17.9 \cdot D^{4}\left(\max \left\{\log b^{\prime}+0.38, \frac{30}{D}, 1\right\}\right)^{2} \log A_{1} \log A_{2} .
$$

In order to apply Lemma ??, we rewrite $\Lambda$, ( see (??)), into the form

$$
\Lambda=\log \left(\alpha^{\Delta} \cdot \sqrt{\frac{b}{a}}\right)-k \log \left(\frac{\beta}{\alpha}\right) .
$$

Hence, we take

$$
D=4, \quad b_{2}=1, \quad b_{1}=k, \quad \alpha_{2}=\alpha^{\Delta} \cdot \sqrt{\frac{b}{a}}, \quad \alpha_{1}=\frac{\beta}{\alpha} .
$$

If $\alpha_{1}$ and $\alpha_{2}$ are multiplicatively dependent, then we have $\alpha_{1}^{p}=\alpha_{2}^{q}$ with nonzero integers $p$ and $q$. Without loss of generality, we suppose that $p>0$. This implies $a^{q} \beta^{2 p}=b^{q} \alpha^{2 p+2 \Delta q}$. Define that $\alpha^{j}=V_{j}+U_{j} \sqrt{a}$ and $\beta^{k}=T_{k}+W_{k} \sqrt{b}$. Combining with the fact $V_{j}, U_{j}, T_{k}$ and $W_{k}$ are both integers and equality

$$
a^{q}\left(T_{2 p}+W_{2 p} \sqrt{b}\right)=b^{q}\left(V_{2 p+2 \Delta q}+U_{2 p+2 \Delta q} \sqrt{a}\right)
$$

we get

It follows that

$$
a^{q} T_{2 p}=b^{q} V_{2 p+2 \Delta q}, \quad a^{q} W_{2 p} \sqrt{b}=b^{q} U_{2 p+2 \Delta q} \sqrt{a} .
$$

$$
a^{2 q}=a^{2 q}\left(T_{2 p}^{2}-b W_{2 p}^{2}\right)=b^{2 q}\left(V_{2 p+2 \Delta q}^{2}-a U_{2 p+2 \Delta q}^{2}\right)=b^{2 q} .
$$


It results $a=b$, which is a contradiction. So $\alpha_{1}$ and $\alpha_{2}$ are multiplicatively independent. It is easy to see that $h\left(\alpha^{\Delta}\right)=\frac{1}{2} \Delta \log \alpha$ and

$h\left(\sqrt{\frac{b}{a}}\right)=\frac{1}{2} \log b=\frac{1}{2} \log \left(n^{2}-1\right)<\frac{1}{2} \log \left((2 m-1)^{2}-1\right)<\log (2 m-1)<\log \alpha$.

Thus we have

$$
h\left(\alpha_{2}\right)=h\left(\alpha^{\Delta} \cdot \sqrt{\frac{b}{a}}\right) \leq h\left(\alpha^{\Delta}\right)+h\left(\sqrt{\frac{b}{a}}\right)<\frac{1}{2}(\Delta+2) \log \alpha=: \log A_{2} .
$$

Moreover, $\gamma=\frac{n+\sqrt{n^{2}-1}}{m+\sqrt{m^{2}-1}}$ is a root of

$$
X^{4}-4 m n X^{3}+\left(4 m^{2}+4 n^{2}-2\right) X^{2}-4 m n X+1 .
$$

The absolute values of its conjugates greater than 1 are $\beta / \alpha$ and $\alpha \beta$. Hence

$$
\log A_{1}:=h(\gamma)=\frac{1}{4}(\log (\beta / \alpha)+\log (\alpha \beta))=\frac{1}{2} \log \beta .
$$

We assume from now and on that $m^{1-\varepsilon} \geq 10^{5}$. We have $\beta=n+\sqrt{n^{2}-1}>$ $2 m \geq 2 m^{1-\varepsilon} \geq 2 \cdot 10^{5}$. This implies $\frac{\left|b_{2}\right|}{D h_{1}}=\frac{1}{2 \log \beta}<0.041$. This leads to

$$
b^{\prime}=\frac{k}{2(\Delta+2) \log \alpha}+0.041 .
$$

Notice that $\Delta=j-k$ is a positive integer. From Lemma ??, we have

$$
\frac{k-1}{2(\Delta+2) \log \alpha}>\frac{0.99 \Delta m^{1-\varepsilon} \log \alpha}{2(\Delta+2) \log \alpha} \geq 0.165 m^{1-\varepsilon} \text {. }
$$

This implies $\log b^{\prime}+0.38 \geq 10>30 / D$. Therefore, by Lemma ?? we obtain

$$
\log |\Lambda| \geq-17.9 \cdot 4^{4}\left(\log b^{\prime}+0.38\right)^{2} \cdot \frac{1}{2} \log \beta \cdot \frac{1}{2}(\Delta+2) \log \alpha .
$$

On the other hand, from Lemma ??, we get

$$
\log |\Lambda|<\log \left(\frac{\alpha^{2}}{\alpha^{2}-1}\right)-2 j \log \alpha<\frac{1}{\alpha^{2}-1}-2 j \log \alpha .
$$

Combining (??) and (??), we have

$$
\frac{j}{\log \beta}<\frac{1}{2\left(\alpha^{2}-1\right) \log \alpha \log \beta}+17.9 \cdot 32\left(\log b^{\prime}+0.38\right)^{2}(\Delta+2) .
$$

It is easy to show that $j \log \alpha>(k-1) \log \beta$, then above inequality imply

$$
\frac{k}{2(\Delta+2) \log \alpha}-0.014<\frac{k-1}{2(\Delta+2) \log \alpha}<0.001+17.9 \cdot 16\left(\log b^{\prime}+0.38\right)^{2} .
$$

It follows that

$$
b^{\prime}<0.055+286.4\left(\log b^{\prime}+0.38\right)^{2} .
$$

We calculate that $b^{\prime}<33380$. Therefore, by (??) we get

$$
k<66760(\Delta+2) \log \alpha .
$$


Combining this and $k-1 \geq 0.99 \Delta m^{1-\varepsilon} \log \alpha$ (see Lemma ??), we obtain

$$
m^{1-\varepsilon}<\frac{66760}{0.99} \cdot \frac{\Delta+2}{\Delta} \leq \frac{66760}{0.99} \cdot 3<202304 .
$$

Thus, we have $m<202304^{\frac{1}{1-\varepsilon}}$. This completes the proof of Theorem ??.

\section{Proof of Theorem ??}

The Diophantine approximation algorithm called the Baker-Davenport reduction method, is used in many papers. The following lemma is a slight modification of the original version of Baker-Davenport reduction method. (See [?, Lemma $5 \mathrm{a}])$.

Lemma 6. Let $M$ be a positive integer and let $\delta, \mu, A$ and $B$ be real numbers with $A>0$ and $B>1$. Assume that $p / q$ be the convergent of the continued fraction expansion of $\delta$ such that $q>6 M$ and let

$$
\eta=\|\mu q\|-M \cdot\|\delta q\|
$$

where $\|\cdot\|$ denotes the distance from the nearest integer. If $\eta>0$, then there is no solution of the inequality

$$
0<j \delta-k+\mu<A B^{-j}
$$

in integers $j$ and $k$ with

$$
\frac{\log (A q / \eta)}{\log B} \leq j \leq M
$$

For any fixed $\varepsilon \in(0,1)$, we only need to check that these cases are determined by $2 \leq m \leq 202304^{\frac{1}{1-\varepsilon}}$. Since $n$ satisfies $m<n<m+m^{\varepsilon}$, then there are about

$$
\begin{gathered}
N(\varepsilon)=\sum_{m=2}^{202304^{1 /(1-\varepsilon)}} \sum_{n=m+1}^{m+m^{\varepsilon}} 1 \leq \int_{1}^{202304^{1 /(1-\varepsilon)}} \int_{t}^{t+t^{\varepsilon}} d v d t \\
=\int_{1}^{202304^{1 /(1-\varepsilon)}} t^{\varepsilon} d t=\left.\frac{t^{1+\varepsilon}}{1+\varepsilon}\right|_{1} ^{202304^{1 /(1-\varepsilon)}} \leq \frac{1}{1+\varepsilon} 202304^{\frac{1+\varepsilon}{1-\varepsilon}}
\end{gathered}
$$

pairs $(m, n)$.

When we choose $\varepsilon=1 / 5$, then $m<4290478$ and $N(\varepsilon)<7.6 \cdot 10^{7}$. This shows (see equation (11) of [?]) that

$$
\frac{j}{\log (e j)}<4.26 \times 10^{13} \log ^{2} \beta .
$$

In our case, we have $j<10^{18}$. By Lemma ?? we may apply Lemma ?? with

$$
\delta=\frac{\log \alpha}{\log \beta}, \quad \mu=\frac{\log \left(\sqrt{\frac{b}{a}}\right)}{\log \beta}, \quad A=\frac{\alpha^{2}}{\left(\alpha^{2}-1\right) \log \beta}, \quad B=\alpha^{2}
$$

and $M=10^{18}$.

The program was developed in PARI/GP running with 200 digits. For the computations, if the first convergent such that $q>6 M$ does not satisfy the condition $\eta>0$, then we use the next convergent until we find the one that 
satisfies the condition. We checked in the ranges $2 \leq m \leq 4290478$ and $m+1 \leq$ $n<m+m^{1 / 5}$.

All the computations were done in about 18 hours. The use of the second convergent was needed in 6147425 cases $(5.57 \%)$, the third convergent was used in 986330 cases $(0.26 \%)$, etc., the 11 th was needed only in $(m, n)=(1219283,1219292)$. In all cases we obtained $j \leq 18$. By Lemma ??, if $m \geq 6$, then we have

$$
0.99 m^{4 / 5} \log \left(m+\sqrt{m^{2}-1}\right)<k-1<j-1 \leq 17 .
$$

This implies $m \leq 8$. In this range, $n$ has to be $m+1$ with the inequalities $m<n<m+m^{1 / 5}$. One can refer to Theorem 4 of [?], there is only the solution $j=k=1$. Thus, in any case there is no positive integer solution with $j k>1$. This completes the proof of Theorem ??.

\section{An application to a family of Thue equations with two PARAMETERS}

We consider the following two-parametric family of Thue equations (11) $X^{4}-4 m n X^{3} Y+\left(4 m^{2}+4 n^{2}-2\right) X^{2} Y^{2}-4 m n X Y^{3}+Y^{4}=1, \quad 1<m<n$, in unknown integers $X$ and $Y$. As a corollary of Theorems ?? and ??, we have:

Corollary 1. If $m<n \leq m+m^{\varepsilon}$, for some real number $\varepsilon \in(0,1)$, then the Thue equation (??) has only integer solutions $(X, Y)=(0, \pm 1),( \pm 1,0)$, for $m>202304^{\frac{1}{1-\varepsilon}}$. Furthermore, for $m<n \leq m+m^{\frac{1}{5}}$, the Thue equation (??) has only integer solutions $(X, Y)=(0, \pm 1),( \pm 1,0)$.

Proof. Let $(X, Y)$ be an arbitrary, but fixed solution. On setting

$$
x=m X^{2}-2 n X Y+m Y^{2}, \quad y=\left|X^{2}-Y^{2}\right|, \quad z=n X^{2}-2 m X Y+n Y^{2},
$$

we have the system of equations (??), so Theorems ?? and ?? complete the proof since $1=y=\left|X^{2}-Y^{2}\right|$ implies that $(X, Y)=( \pm 1,0),(0, \pm 1)$.

In 2005, Jadrijević [?]-[?] studied the two-parametric family of Thue equations

$$
x^{4}-2 m n x^{3} y+2\left(m^{2}-n^{2}+1\right) x^{2} y^{2}+2 m n x y^{3}+y^{4}=1
$$

and transformed it into the system of Pell equations

$$
V^{2}-\left(m^{2}+2\right) U^{2}=-2, \quad Z^{2}-\left(n^{2}-2\right) U^{2}=2 .
$$

She proved that for every $0.5<\varepsilon \leq 1$, there exists an effectively computable constant $C(\varepsilon)$ such that if $m \neq 0, \max \{|m|,|n|\} \geq C(\varepsilon)$ and $\operatorname{gcd}(m, n) \geq$ $\max \left\{|m|^{\varepsilon},|n|^{\varepsilon}\right\}$, then the system of Pell equations (??) has only the trivial solutions $(V, Z, U)=( \pm m, \pm n, 1)$, and gave some particular values of $C(\varepsilon)$. Further, she proved that this family of Thue equations has no solutions if $\operatorname{gcd}(x y, m n)=1$ and $x y \neq 0$.

Using a little more work and a method similar to that above, one can obtain the following result.

Theorem 3. If $|m-n| \leq \max \{m, n\}^{\varepsilon}, \varepsilon \in(0,1)$, then the system of Pell equations (??) has only integer solution $(V, Z, U)=( \pm m, \pm n, 1)$, for $\max \{m, n\} \geq$ $C(\varepsilon)$, where $C(\varepsilon)$ is an effectively computable constant depending on $\varepsilon$. 
The outline of proof of Theorem ?? is the following: Transform equations (??) into the intersections of two Lucas sequences such as

$$
y=H_{j}=\frac{\nu^{j}-\bar{\nu}^{j}}{\sqrt{2\left(m^{2}+2\right)}}=K_{k}=\frac{\delta^{k}-\bar{\delta}^{k}}{\sqrt{2\left(n^{2}-2\right)}},
$$

where $j, k$ are odd positive integers and

$$
\nu=\frac{m+\sqrt{m^{2}+2}}{\sqrt{2}}, \bar{\nu}=\frac{m-\sqrt{m^{2}+2}}{\sqrt{2}}, \delta=\frac{n+\sqrt{n^{2}-2}}{\sqrt{2}}, \bar{\delta}=\frac{n-\sqrt{n^{2}-2}}{\sqrt{2}} .
$$

Notice that the shapes of these two Lucas numbers are a little different. In order to deal with equation (??), we need to consider two cases: $m<n<m+m^{\varepsilon^{\prime}}$ and $n<m<n+m^{\varepsilon^{\prime}}$. In the case $n<m$, one can change the order of $j$ and $k$ (also, $\alpha$ and $\beta$ ) and follow the lines of Lemmas ??-??. For the part of the proof related to the linear form in two logarithms, one can easily see that $\frac{\nu}{\delta}$ is a root of

$$
1+2 n m X+\left(2 m^{2}-2 n^{2}+2\right) X^{2}-2 n m X^{3}+X^{4} .
$$

\section{REFERENCES}

[1] W. S. Anglin, Simultaneous Pell equations, Math. Comp. 65 (1996), 355-359.

[2] A. Baker and H. Davenport, The equations $3 x^{2}-2=y^{2}$ and $8 x^{2}-7=z^{2}$, Quart. J. Math. Oxford 20 (1969), 129-137.

[3] M. A. Bennett, Solving families of simultaneous Pell equations, J. Number Theory, 67 (1997), 246-251.

[4] M. A. Bennett, On the number of solutions of simultaneous Pell equations, J. Reine Angew. Math. 498 (1998), 173-199.

[5] M. A. Bennett and P.G. Walsh, Simultaneous quadratic equations with few or no solutions, Indag. Mathem. 11 (2000), 1-12.

[6] M. A. Bennett, M. Cipu, M. Mignotte, and R. Okazaki, On the number of solutions of simultaneous Pell equations II, Acta Arith. 122 (2006), 407-417.

[7] M. Cipu and M. Mignotte, On the number of solutions of simultaneous hyperbolic Diophantine equations, J. Number Theory 125 (2007), 356-392.

[8] L. E. Dickson, History of the theory of numbers. Vol. II: Diophantine analysis, Chelsea Publishing Co., New York, 1966.

[9] A. Dujella and A. Pethö, A generalization of a theorem of Baker and Davenport, Quart. J. Math. Oxford Ser. (2) 49 (1998), 291-306.

[10] B. Jadrijević, A system of Pellian equations and related two-parametric family of quartic Thue equations, Rocky Mountain J. Math. 35 (2005), 547-572.

[11] B. Jadrijević, On two-parametric family of quartic Thue equations, J. Théor. Nombres Bordx. 17 (2005), 171-177.

[12] K. S. Kedlaya, Solving constrained Pell equations, Math. Comp. 67 (1998), 833-842.

[13] M. Laurent, Linear forms in two logarithms and interpolation determinants II, Acta Arith 133 (2008), 325-348.

[14] M. Le, A note on the simultaneous Pell equations $x^{2}-a y^{2}=1$ and $z^{2}-b y^{2}=1$, Glasnik Matematicki, 47 (2012), 53-59.

[15] D. W. Masser, J. H. Rickert, Simultaneous Pell Equations, J. Number Theory, 61 (1996), $52-66$.

[16] K. Ono, Euler's concordant forms, Acta Arith., 78, (1996), 101-123.

[17] C. L. Siegel. Über einige Anwendungen diophantischer Approximationen, Abh. Preuss. Akad. Wiss., (1929), 1.

[18] H. P. Schlickewei, The number of subspaces occuring in the p-adic subspace theorem in Diophantine approximation, J. Reine Angew. Math. 406 (1990), 44-108. 
[19] L. Szalay, On the resolution of simultaneous Pell equations, Annales Math. et Info. 34 (2007), 77-87.

[20] A. Thue. Über Annäherungenswerte algebraischen Zahlen, J. reine angew. Math. 135 (1909), 284-305.

[21] P. Z. Yuan, On the number of solutions of simultaneous Pell equations, Acta Arith. 101 (2002), 215-221.

[22] P. Z. Yuan, Simultaneous Pell equations, Acta Arith. 115 (2004), 119-132.

Bo He

Department of Mathematics

Aba Teacher's College

Wenchuan, Sichuan 623000

P. R. China

bhe@live.cn

Ákos Pintér

Institute of Mathematics, MTA-DE Research Group

"Equations, Functions and Curves"

Hungarian Academy of Sciences and University of Debrecen

P. O. Box 12, H-4010 Debrecen, Hungary

apinter@science.unideb.hu

\author{
Alain Togbé \\ Department of Mathematics \\ Purdue University North Central \\ 1401 S. U.S. 421 \\ Westville, IN 46391 USA \\ atogbe@pnc.edu
}

